\title{
Modelling of a vibro-impact power take-off mechanism for wave energy conversion
}

\author{
Bingyong Guo and John V. Ringwood
}

\begin{abstract}
Ocean wave spectra are broad-banded, while wave energy conversion systems are designed to harvest energy efficiently within a narrow frequency range, resulting in low overall power capture efficiency. This paper aims to introduce a nonlinear vibro-impact power take-off (PTO) mechanism for a heaving point absorber, in order to broaden its power capture bandwidth and provide end-stop function. A mathematical model of the vibro-impact mechanism is derived, and numerical simulations are conducted under regular wave conditions, to investigate the effect of the vibro-impact mechanism on the system performance. Numerical results illustrate that the heaving point absorber with the vibro-impact PTO system exhibits a characteristics of band-pass effect, and the nonlinear vibro-impact mechanism has the potential to broaden power capture bandwidth, achieve end-stop function, and attenuate peak-to-average power ratio.
\end{abstract}

\section{INTRODUCTION}

Ocean waves are generally characterised by broad-banded spectra, and wave spectra vary from time to time [1], [2]. Current wave energy converters (WECs) are typically designed to harvest energy efficiently within a narrow frequency range close to resonance. In order to harvest wave energy more efficiently with time-varying wave frequency, a range of control strategies [3] have been developed and applied to maximise power absorption of WECs across a broad frequency range. Alternatively, novel design in WEC systems or components can also broaden the power capture bandwidth. For instance, some novel concepts in power takeoff (PTO) design have the potential to achieve high power absorption. A 'negative' spring, termed WaveSpring, was developed and tested by CorPower Ocean to increase WEC system's response bandwidth [4], whose principle was the same as the bistable mechanical system with two symmetrically oblique springs to achieve a snap-through mechanism [5]. In addition to nonlinear springs, nonlinear dampers were studied numerically and experimentally in [6]. These studies conclude that a properly designed nonlinear mechanism in the PTO system has the potential to enhance average power absorption of WECs.

This work proposes a nonlinear vibro-impact PTO mechanism integrated inside a heaving point absorber. As shown in Fig. 1, a floating buoy $\left(M_{b}\right)$ oscillates under the excitation of incident waves. An inner mass $\left(M_{m}\right)$ is connected to the buoy by a primary spring $\left(k_{1}\right)$ and a linear damper $(c)$, and oscillates accordingly due to the interaction force with the floater. The key mechanism is that the secondary

Bingyong Guo and John V. Ringwood are with the Centre for Ocean Energy Research, Department of Electronic Engineering, Maynooth University, Maynooth, Ireland, Bingyong.Guo@mu.ie, John. Ringwood@mu.ie. $\left(k_{2}\right)$ and tertiary springs $\left(k_{3}\right)$ attached to the top ceiling and floor of the buoy provide upper or lower impacts when the relative displacement between the inner mass and the buoy exceeds the gaps $G_{1}$ or $G_{2}$ (positive parameters defined at the equilibrium point), respectively. Heaving point absorbers are typically characterised by large peak-to-average power ratios, which can be up to 26.9 in sea testing [7]. A large peak-toaverage power ratio introduces challenges to the design of highly-efficient and cost-effective PTO devices, while also producing poor power quality at the grid connection point due to large power fluctuations and, hence, extra efforts are required to smooth the generated power by energy storage or control [8]. In addition, end-stop function is required for point absorbers, and can be achieved by control [9] or mechanical design [10]. In this study, the effect of the vibroimpact mechanism on the peak-to-average power ratio and end-stop function will be outlined.

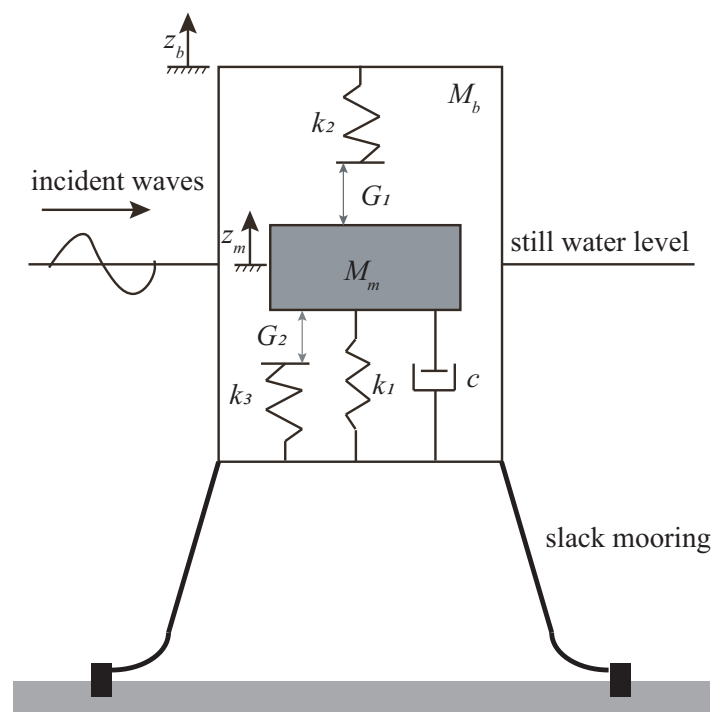

Fig. 1. Schematic diagram of a heaving point absorber integrated with a vibro-impact PTO mechanism. The total mass of the buoy and the inner mass is constant to set the buoy semi-submerged. The inner mass is kept at buoy's centre by adjusting the initial length of the supporting spring.

In this paper, a mathematical model for the vibro-impact PTO is derived and numerical simulations are conducted to investigate how the design parameters, i.e. the inner mass and spring stiffness, and initial conditions, influence the WEC's dynamics and power absorption in regular waves. Irregular waves are not considered here as they will introduce extra complexity to the nonlinear dynamics of the vibro-impact mechanism. The hydrodynamic properties of the wave-buoy 
interaction is computed by a boundary element method code $\mathrm{NEMOH} \mathrm{[11].} \mathrm{System} \mathrm{identification} \mathrm{techniques} \mathrm{are} \mathrm{then}$ applied to approximate the radiation and excitation forces so that a piece-wise linear model can be derived. Numerical simulations demonstrate: (i) The response amplitude operator (RAO) of the relative motion between the buoy and the inner mass acts like a 'band-pass filter', with relatively small gain values when the wave frequency is low. As extreme sea states are characterised by low frequency, the vibroimpact PTO system is inherently decoupled from extreme waves and shows high reliability and survivability. (ii) The vibro-impact events have the capability to enhance the point absorber's power capture bandwidth and provide end-stop function, simultaneously. (iii) The vibro-impact mechanism may introduce rich and complex nonlinear dynamics, especially when impacts occur. Hence, the system dynamics are sensitive to the design parameters, wave frequency and initial conditions.

The remainder of the paper is structured as follows. Section II details the mathematical modelling of the heaving point absorber with the vibro-impact PTO device. Parametric studies and numerical results are discussed in Sections III$\mathrm{V}$ by varying the inner mass, secondary/tertiary stiffness and initial conditions, respectively. Conclusions are drawn in Section VI.

\section{MODELling OF VIBRO-IMPACT POWER TAKE-OFF MECHANISM}

In this section, for the heaving point absorber with vibroimpact PTO system shown in Fig. 1, a mathematical model is derived for numerical simulation, including the buoy-mass and wave-buoy interactions.

\section{A. Equation of motion}

From Fig. 1, the motion of the buoy is described by

$$
M_{b} \ddot{z}_{b}=f_{e}+f_{r}+f_{h s}+f_{i},
$$

where $f_{e}, f_{r}$ and $f_{h s}$ represent the excitation, radiation and hydrostatic forces, respectively. $f_{i}$ represents the interaction force between the buoy and the inner mass. $z_{b}$ is the heaving displacement of the buoy, $\ddot{z}_{b}$ represents its acceleration, and $M_{b}$ is the floater's mass. For simplicity, only the heave motion is investigated with its positive direction defined upward, and the mooring and viscous forces are omitted here.

For the inner mass, its equation of motion is written as

$$
M_{m} \ddot{z}_{m}=-f_{i},
$$

where $M_{m}, z_{m}$ and $\ddot{z}_{m}$ represent the inner mass, its displacement and acceleration in heave, respectively. The interaction force $f_{i}$ depends on the relative displacement between the inner mass and the buoy, with three possible cases: (i) When the relative displacement is within the two gaps, only the primary spring with stiffness $k_{1}$ and the PTO damper with coefficient $c$ are active. (ii) When the relative displacement is positive and larger than the upper gap $G_{1}$, the secondary spring with stiffness $k_{2}$ is active. (iii) When the relative displacement exceeds the lower gap $G_{2}$, the tertiary spring with stiffness $k_{3}$ is active. Therefore, the interaction force can be summarised as

$$
f_{i}= \begin{cases}k_{1} z_{r}+k_{2}\left(z_{r}-G_{1}\right)+c v_{r}, & z_{r} \geq G_{1}, \\ k_{1} z_{r}+c v_{r}, & G_{1}>z_{r}>-G_{2}, \\ k_{1} z_{r}+k_{3}\left(z_{r}+G_{2}\right)+c v_{r}, & z_{r} \leq-G_{2},\end{cases}
$$

where $z_{r}=z_{m}-z_{b}$ and $v_{r}=\dot{z}_{r}$ represent the relative displacement and velocity, respectively. The PTO force is written as

$$
f_{\text {pto }}=c v_{r} .
$$

\section{B. Wave-buoy interaction}

For a vertical cylinder, the hydrostatic force is given as

$$
f_{h s}=-\rho g \pi r^{2} z_{b},
$$

where $\rho, g$ and $r$ are the water density, gravity constant and buoy radius, respectively. The radiation force, in the timedomain, is given as

$$
f_{r}=-m_{\infty} \ddot{z}_{b}-k_{r} * \dot{z}_{b}
$$

where $m_{\infty}$ is the added mass at infinite frequency and $k_{r}$ is the impulse response function (IRF) of the radiation force. The symbol $*$ represents the convolution operator. Finite order approximations of the radiation force were studied in [12], [13]. Hence, the convolution term $f_{r c}=k_{r} * \dot{z}_{b}$ can be approximated by

$$
\begin{aligned}
\dot{x}_{r} & =A_{r} x_{r}+B_{r} \dot{z}_{b}, \\
f_{r c} & \approx C_{r} x_{r},
\end{aligned}
$$

where $x_{r} \in \mathbb{R}^{n \times 1}$ is the state vector for the identified system. $A_{r} \in \mathbb{R}^{n \times n}, B_{r} \in \mathbb{R}^{n \times 1}, C_{r} \in \mathbb{R}^{1 \times n}$ are the system matrices, and $n$ is the dynamical order, given in the Appendix.

The excitation force $f_{e}$ can be determined quantitatively by its frequency response function (FRF), represented as

$$
F_{e}(j \omega)=H_{e}(j \omega) A(j \omega),
$$

where $H_{e}(j \omega)$ is the FRF of the excitation force and $A(j \omega)$ is the frequency-domain representation of incident wave $\eta(t)$. In the time-domain, the excitation force is expressed as

$$
f_{e}(t)=k_{e}(t) * \eta(t)=\int_{-\infty}^{\infty} k_{e}(t-\tau) \eta(\tau) d \tau
$$

where $k_{e}(t)=\frac{1}{2 \pi} \int_{-\infty}^{\infty} H_{e}(j \omega) e^{j \omega t} d \omega$ is the excitation force IRF, with $\omega$ representing wave frequency. Unfortunately, $k_{e}(t)$ is noncausal [14] and its causalisation was studied in [15], [16]. The causalised system with wave prediction gives the same excitation force as the noncausal system, since

$$
f_{e}(t)=k_{e}(t) * \eta(t)=k_{e, c}(t) * \eta_{p}(t),
$$

where $k_{e, c}(t)=k_{e}\left(t-t_{c}\right)$ and $\eta_{p}(t)=\eta\left(t+t_{c}\right)$ are the causalised IRF of the excitation force and the predicted wave elevation advanced by $t_{c}$, respectively. $t_{c}$ is the causalisation time defined in [15], [16]. Therefore, the excitation force can be approximated by

$$
\begin{aligned}
\dot{x}_{e} & =A_{e} x_{e}+B_{e} \eta_{p}, \\
f_{e} & \approx C_{e} x_{e}+D_{e} \eta_{p}
\end{aligned}
$$


where $x_{e} \in \mathbb{R}^{n \times 1}$ is the state vector for the excitation system. $A_{e} \in \mathbb{R}^{n \times n}, B_{e} \in \mathbb{R}^{n \times 1}, C_{e} \in \mathbb{R}^{1 \times n}$ and $D_{e} \in \mathbb{R}^{1 \times 1}$ are the system matrices, and $n$ is the order, given in the Appendix.

In this work, a general cylindrical buoy, as shown in Fig. 1, is utilised, of $r=1 \mathrm{~m}$ in radius, $h=2 \mathrm{~m}$ in height, and $d=1 \mathrm{~m}$ in draft, and the motion is constrained to heave only. The hydrodynamic coefficients are computed via NEMOH [11]. The numerical and identified IRFs of the radiation and excitation forces are compared in Fig. 2.
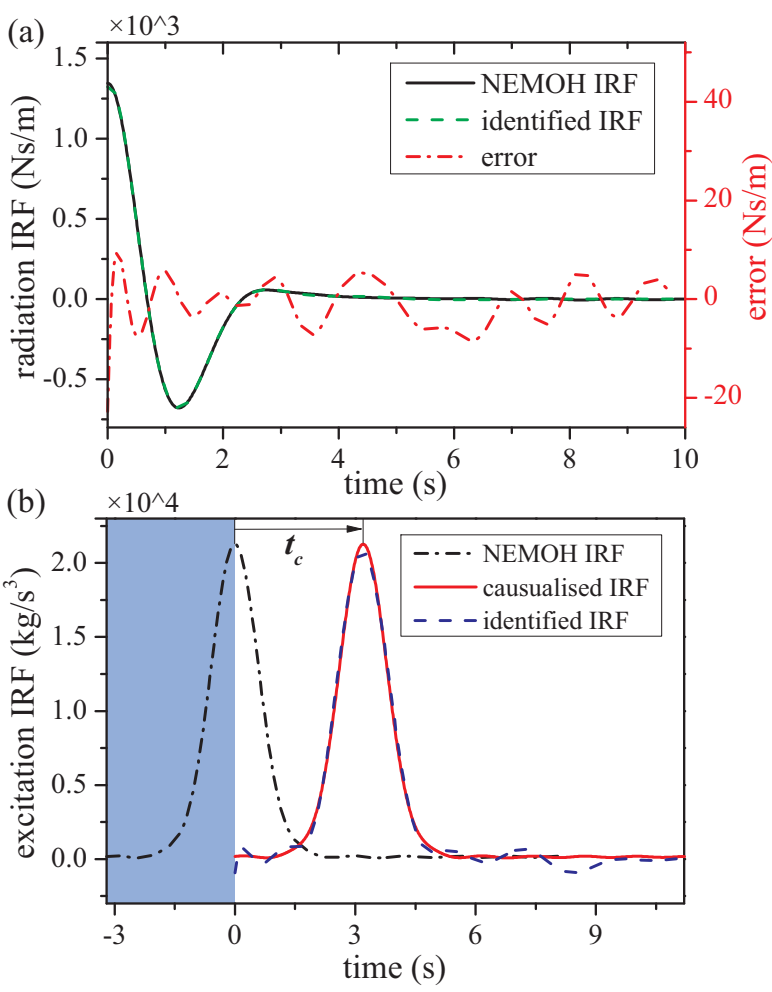

Fig. 2. Comparison of IRFs of (a) the radiation and (b) excitation forces. The excitation FRF is obtained via NEMOH and then used to compute its IRF.

\section{INFLUENCE OF INNER MASS}

The nonlinear dynamics of the vibro-impact PTO are sensitive to design parameters, including $M_{m}, k_{1}, k_{2}, k_{3}, G_{1}$, $G_{2}$, and $c$, and wave conditions in terms of wave height $H$ and frequency $\omega$. In this section, a parametric study is conducted by varying the inner mass from $200 \mathrm{~kg}$ to 3000 $\mathrm{kg}$, subjected to harmonic waves with height $H=0.8 \mathrm{~m}$ and frequency $\omega$ varying from $0.06 \mathrm{rad} / \mathrm{s}$ to $6.28 \mathrm{rad} / \mathrm{s}$. To evaluate the dynamics of the heaving point absorber with vibro-impact PTO, the relative RAO is defined as

$$
R A O_{r}=\frac{\max \left|z_{r}\right|}{H / 2}
$$

To evaluate the performance of the vibro-impact PTO mechanism, the instantaneous power $P_{i}$, average power $P_{a}$ and peak-to-average power ratio $P_{p 2 a}$ are defined as

$$
\begin{aligned}
P_{i} & =c v_{r}^{2}, \\
P_{a} & =\frac{1}{T} \int_{0}^{T} P_{i} d t, \\
P_{p 2 a} & =\frac{\max \left(P_{i}\right)}{P_{a}},
\end{aligned}
$$

where $T$ is the wave period.

The relative RAO between the inner mass and the buoy is given in Fig. 3, considering a wide range of inner mass variation and wave frequency fluctuation. In Fig. 3(a), wave frequency increases from $0.06 \mathrm{rad} / \mathrm{s}$ to $6.28 \mathrm{rad} / \mathrm{s}$, while it decreases from $6.28 \mathrm{rad} / \mathrm{s}$ to $0.06 \mathrm{rad} / \mathrm{s}$ in Fig. 3(b). Both Figs. 3(a) and (b) indicate that: (i) The relative RAO has a characteristic typical of a band-pass filter, with a small gain when $\omega<1 \mathrm{rad} / \mathrm{s}$ or $\omega>3.5 \mathrm{rad} / \mathrm{s}$, and a large gain when the wave frequency is close to the resonance frequency region. The maximum relative RAO rises to 4.5 in Fig. 3(a). (ii) The resonance frequency decreases from 2.5 $\mathrm{rad} / \mathrm{s}$ to $1.5 \mathrm{rad} / \mathrm{s}$ as the inner mass increases from $200 \mathrm{~kg}$ to $3000 \mathrm{~kg}$. That is, a larger inner mass results in a lower resonance frequency. (iii) When the wave frequency is close to the resonance frequency region (especially in the areas marked by red solid/dash rectangles), the relative RAO in Fig. 3(a) is much larger than its counterpart in Fig. 3(b), indicating the existence of multistability. Detailed discussion of multistability is given in Section V.
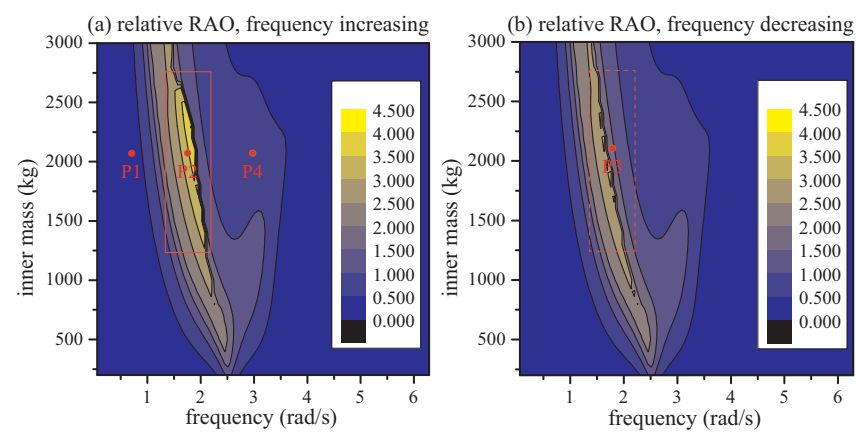

Fig. 3. Comparison of relative RAO under a wide range of inner mass variation as the wave frequency increases in (a), and decreases in (b). The results in the red solid rectangle are larger than their counterparts in the red dash rectangle. Four points $\mathrm{P} 1-\mathrm{P} 4$ are marked by red dots and their time traces are compared in Fig. 5. In simulation, $k_{2}=k_{3}=20000 \mathrm{~N} / \mathrm{m}$ with other parameters given in the Appendix.

In this work, the performance of the vibro-impact PTO mechanism is evaluated by the average power $P_{a}$ and peakto-average power ratio $P_{p 2 a}$, defined in Eqs. (16) and (17), respectively. Under a wide range of the inner mass variation, contour plots of the average power and peak-to-average power ratio are given in Fig. 4, with wave frequency increasing in Figs. 4(a)-(b), and decreasing in Figs. 4(c)-(d). Fig. 4 illustrates that: (i) Figs. 4(a) and (c) show the band-pass characteristics of the average power, with very low power output at a low or high frequency, and with high power output when the wave frequency is close to the device's resonant frequency. (ii) In Figs. 4(b) and (d), the peak-to-average 
power ratio is small, varying from 1.5 to $3.5\left(P_{p 2 a}=2\right.$ for AC power). Hence, the vibro-impact PTO mechanism can either enlarge or reduce slightly the peak-to-average power ratio, relying on the design parameters and wave conditions. (iii) The maximum values of average power in Figs. 4(a) and (c) do not necessarily indicate maximum values of peak-toaverage power ratio in Figs. 4(b) and (d), respectively. (iv) Figs. 4(a) and (c) show a high accordance with Figs. 3(a) and (b), respectively. That is, a larger relative RAO also means a larger average power capture. (v) Comparing Figs. 4(a)(b) with Figs. 4(c)-(d) correspondingly, it also indicates that the performance of the vibro-impact PTO is sensitive to initial conditions due to multistability. Detailed discussion of multistability is given in Section V.
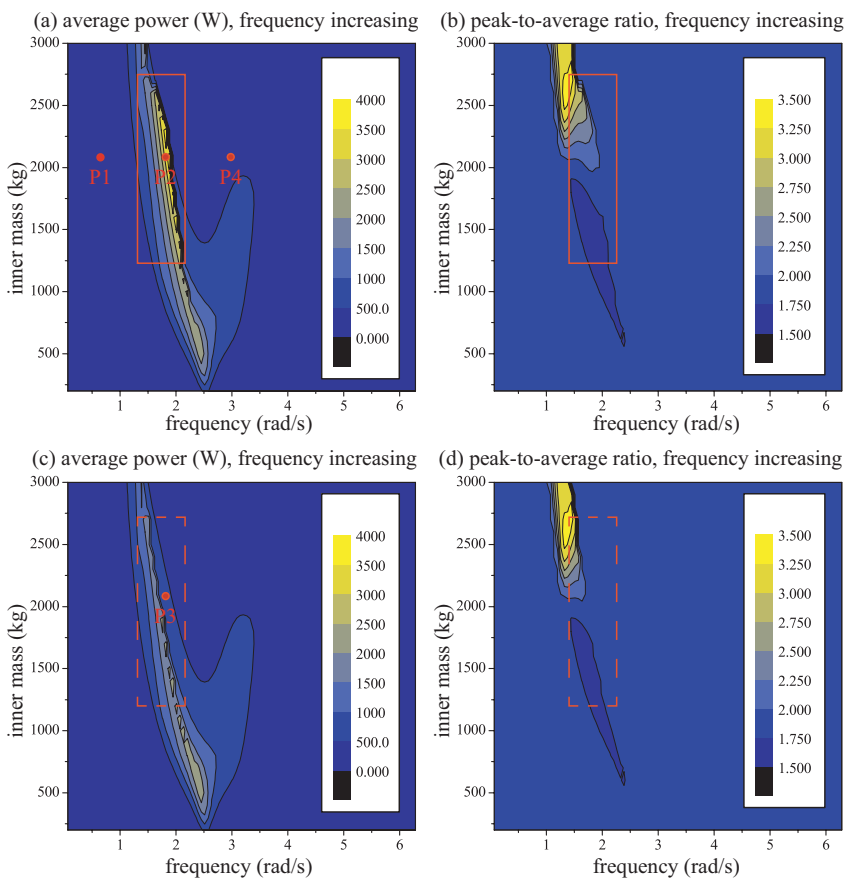

Fig. 4. Contour plots of the average power and peak-to-average power ratio with wave frequency increasing in (a)-(b), and decreasing in (c)-(d). The results in the red sold rectangles are larger than their counterparts in the red dash rectangles, and the time series of the red dots marked P1-P4 are compared in Fig. 5. In simulation, $k_{2}=k_{3}=20000 \mathrm{~N} / \mathrm{m}$ with other parameters given in the Appendix.

The relative motion is typical of band-pass characteristics, which can be an advantage of the vibro-impact PTO mechanism to match a specific wave spectrum via design optimisation. It also can be used to improve device survivability under extreme sea states, as large waves normally occur at low frequency and the vibro-impact PTO system is automatically detuned from extreme waves. To address the band-pass effect and multistability of the vibro-impact PTO device, four simulation conditions marked by red dots P1-P4 in Figs. 3 and 4 are selected to compare their time traces in Fig. 5, with simulation conditions of $\omega=0.8 \mathrm{rad} / \mathrm{s}$ for Fig. 5(a), $\omega=$ $1.9 \mathrm{rad} / \mathrm{s}$ for Figs. 5(b)-(c), and $\omega=3 \mathrm{rad} / \mathrm{s}$ Fig. 5(d). The inner mass is $M_{m}=2100 \mathrm{~kg}$ and the impact springs' stifffness is $k_{2}=k_{3}=20000 \mathrm{~N} / \mathrm{s}$ with other simulation conditions given in the Appendix. Zero initial conditions are applied in Figs. 5(a), (c) and (d), while the initial condition for Fig. 5(b) is $\left[z_{b 0}, \dot{z}_{b 0}, z_{m 0}, \dot{z}_{m 0}\right]^{\prime}=[-0.5,0,0.5,5]^{\prime}$. In Fig. 5(a), the buoy, inner mass and relative displacements are small and, consequently, the instantaneous and average power outputs are small. The average power is less than $1 \mathrm{~W}$, which means that the vibro-impact PTO mechanism is inherently decoupled from waves of low frequency. This shows that the vibroimpact PTO has the potential to improve its survivability and reliability under extreme sea states which is typically characterised by low frequency. Comparing the results in Figs. 5(b) and (c), the dynamics and performance of the vibro-impact PTO system are sensitive to initial conditions due to multistability. In Fig. 5(d), the buoy and inner mass motions are small, but the relative motion is slightly larger as there exists a large phase shift between motions of the buoy and the inner mass, resulting in larger instantaneous and average power outputs than those in Fig. 5(a).
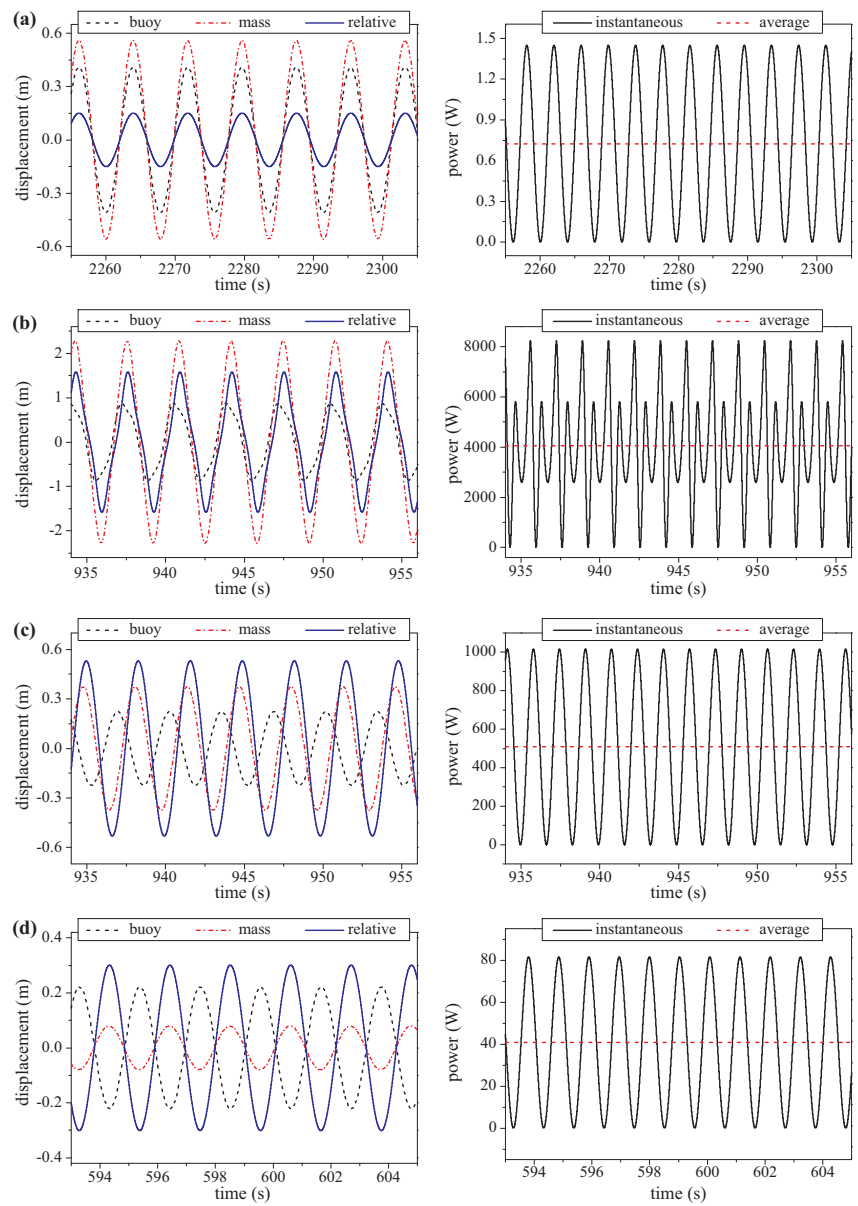

Fig. 5. Time traces of the buoy, mass and relative displacements, instantaneous and average power outputs, with (a)-(d) representing the time traces for the points P1-P4 in Figs. 3 and 4, respectively. The simulation conditions are $\omega=0.8 \mathrm{rad} / \mathrm{s}$ for (a), $\omega=1.9 \mathrm{rad} / \mathrm{s}$ for (b)-(c), and $\omega=3 \mathrm{rad} / \mathrm{s}$ for (d), with $M_{m}=2100 \mathrm{~kg}, k_{2}=k_{3}=20000 \mathrm{~N} / \mathrm{m}$ and other parameters given in the Appendix. The initial condition is $\left[z_{b 0}, \dot{z}_{b 0}, z_{m 0}, \dot{z}_{m 0}\right]^{\prime}=[-0.5,0,0.5,5]^{\prime}$ for (b) and zero initial conditions are used for (a), (b) and (d). 


\section{INFLUENCE OF SPRING STIFFNESS}

As shown in Fig. 5(b), the amplitude of the relative displacement is about $1.6 \mathrm{~m}$ while the physical constraint is $1 \mathrm{~m}$ (half the buoy height). Hence, design optimisation is required to limit the relative motion, such as increasing the buoy's height, the stiffness of the secondary and tertiary springs, or decreasing the impact gaps. In this section, the influence of the secondary and tertiary stiffness on the dynamics and performance of the vibro-impact PTO system is discussed.

Fig. 6 illustrates the relative RAO with varying stiffness of the secondary and tertiary springs. The black solid curve represents the simulation results with $k_{2}=k_{3}=0 \mathrm{~N} / \mathrm{m}$, in which vibro-impact events have no effect. The red dash and blue dot curves represent the simulation results with small stiffness of the secondary and tertiary springs at $k_{2}=$ $k_{3}=20000 \mathrm{~N} / \mathrm{m}$ with respect to the frequency increasing and decreasing cases, respectively. The pink dash-dot and olive dash-dot-dot curves represent the simulation results with large stiffness of the secondary and tertiary springs, for $k_{2}=k_{3}=200000 \mathrm{~N} / \mathrm{m}$ with respect to the frequency increasing and decreasing cases, respectively. The physical constraint is given in a cyan short-dash line.

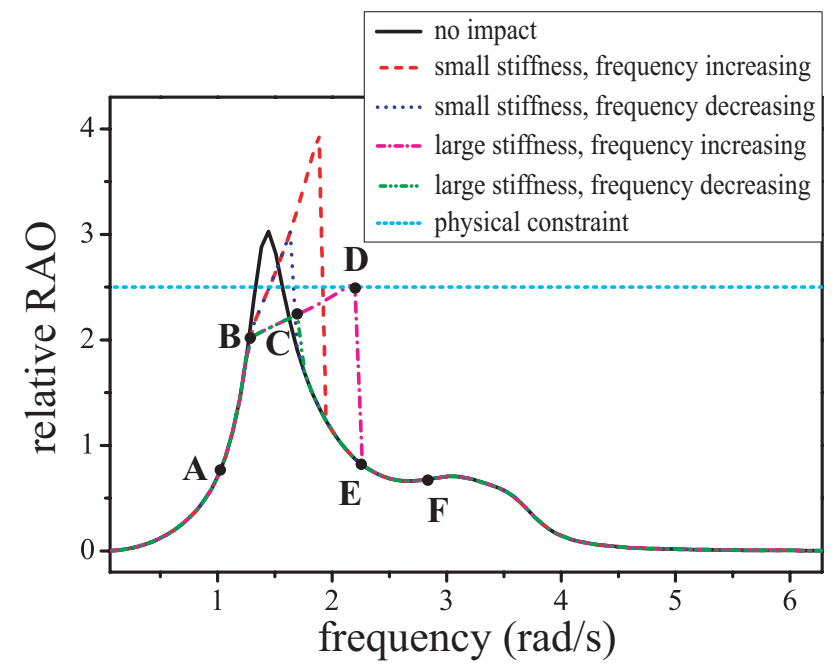

Fig. 6. (a) Relative RAOs with various stiffness of the secondary and tertiary springs. The black solid, red dash, blue dot, pink dash-dot, and olive dash-dot-dot curves represent the results at $k_{2}=k_{3}=0 \mathrm{~N} / \mathrm{m}, k_{2}=$ $k_{3}=20000 \mathrm{~N} / \mathrm{m}$ with increasing and decreasing frequency, and $k_{2}=k_{3}=$ $200000 \mathrm{~N} / \mathrm{m}$ with increasing and decreasing frequency, respectively. The cyan short-dash curve represents the physical constraint. For the simulation, $M_{m}=2100 \mathrm{~kg}$.

As shown in Fig. 6, the trends of relative RAOs show different characteristics when the frequency increases and decreases. For the simulation with relatively large stiffness of $k_{2}=k_{3}=200000 \mathrm{~N} / \mathrm{m}$, the relative RAO's locus is $A \rightarrow B \rightarrow C \rightarrow D \rightarrow E \rightarrow F$ as the frequency increases, while the locus is $F \rightarrow E \rightarrow C \rightarrow B \rightarrow A$ as the frequency decreases. Therefore, multistability exists within the frequency range between the points $\mathrm{C}$ and $\mathrm{E}$. Comparing the red dash curve with the pink dash-dot curve, it can be seen that the relative motion can be constrained effectively by increasing the stiffness of the secondary and tertiary springs. In addition, the bandwidth is broaden by when vibro-impact events occur. Therefore, a larger stiffness value for the secondary and tertiary springs is preferred to limit the relative motion and enlarge the bandwidth, simultaneously.

\section{INFLUENCE OF INITIAL CONDITION}

As discussed in Sections III and IV, the vibro-impact mechanism can introduce multistable phenomenon, for which the system dynamics are sensitive to initial conditions. To investigate the influence of initial conditions on the system dynamics, basins of attraction are computed in this paper, and the results are shown in Fig. 7.
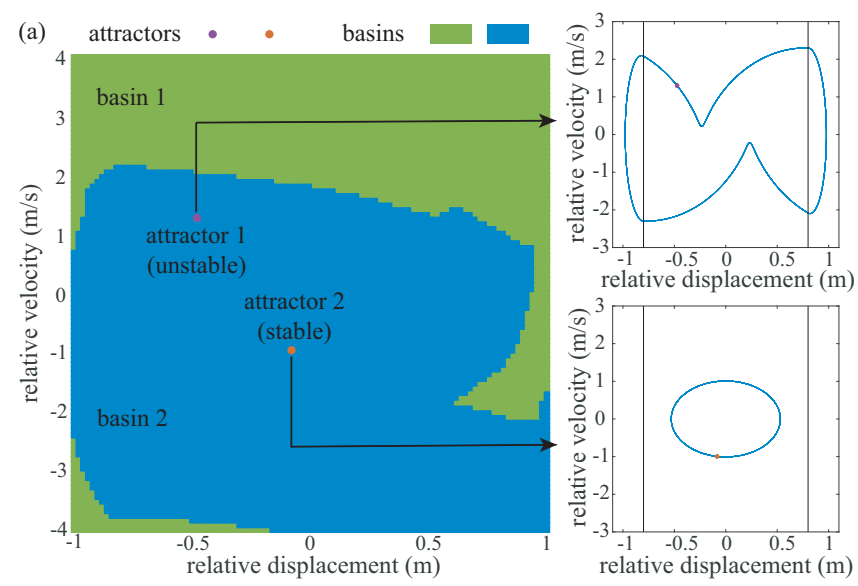

(b)

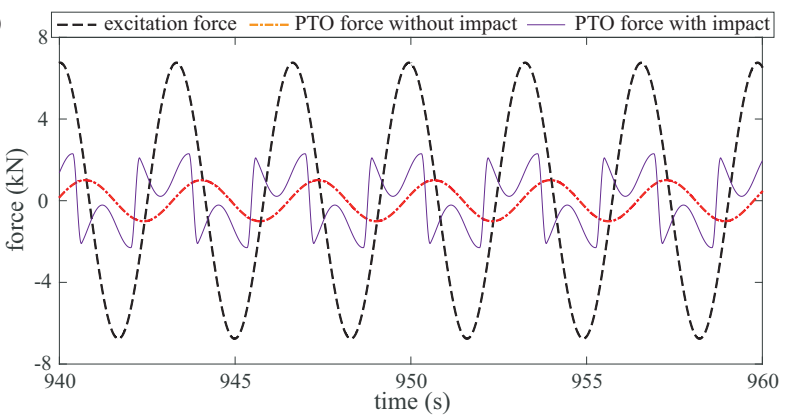

Fig. 7. (a) Basin plots for a broad range of initial conditions with the attractors' phase portraits, and (b) comparison of the PTO force with and without impacts. The simulation conditions are $M_{m}=2100 \mathrm{~kg}, \omega=$ $1.9 \mathrm{rad} / \mathrm{s}$ and $k_{2}=k_{3}=200000 \mathrm{~N} / \mathrm{m}$.

Fig. 7(a) shows that the system dynamics depend significantly on initial conditions. The dynamics for the initial conditions coloured in green converge to the attractor with a larger orbit profile, while the dynamics for the initial conditions coloured in blue converge to the attractor with a smaller orbit profile. Vibro-impact events occur twice per period of excitation for the larger orbit, and no impacts occur for the smaller one. As shown in Fig. 7(b), the PTO force with vibro-impact events is much larger than that without impact, showing that the vibro-impact events can enlarge the amplitude of the PTO force. Meanwhile, the "phase shift' between the PTO and excitation forces is reduced by the vibro-impact occurrence. Therefore, the vibro-impact mechanism has the potential to improve the power capture 
of the WEC system by amplifying the amplitude of the PTO force and reducing 'phase shift' relative to the excitation force.

\section{CONCLUSION}

This study proposes a vibro-impact PTO mechanism for a heaving point absorber, with specific foci on its mathematical model and nonlinear dynamics. Numerical simulations are conducted by varying the inner mass, spring stiffness of the secondary and tertiary springs, wave frequency and initial conditions. Numerical results show that the vibro-impact PTO mechanism is characterised by a band-pass effect and, therefore, it is automatically detuned from extreme waves, normally characterised by low frequencies. Numerical studies also conclude that the peak-to-average power ratio can be either amplified or attenuated slightly by the vibroimpact PTO device, varying from 1.5 to 3.5 , under a wide range of inner mass and wave frequency variations. By increasing the stiffness of the secondary and tertiary springs, the relative motion between the inner mass and the buoy can be constrained effectively. These properties of the vibroimpact PTO mechanism may be useful to improve system survivability under extreme sea states and to enhance power capture bandwidth under moderate sea states.

The nonlinear dynamics of the vibro-impact PTO system are sensitive to the design parameters, and hence design optimisation is required. Ongoing work focuses on parametric studies of the design parameters by varying the inner mass, spring stiffness, impact gaps and PTO damping coefficient over a wide range to provide guidelines for prototype design.

\section{APPENDIX}

Simulation conditions are: buoy radius $r=1 \mathrm{~m}$, height $h=2 \mathrm{~m}$, draft $d=1 \mathrm{~m}$, total mass of the buoy and the inner mass $M_{t}=M_{b}+M_{m}=3220.13 \mathrm{~kg}$, water density $\rho=$ $1025 \mathrm{~kg} / \mathrm{m}^{3}$, gravity constant $g=9.81 \mathrm{~N} / \mathrm{kg}$, added mass at infinite frequency $m_{\infty}=1883.47 \mathrm{~kg}$, primary spring stiffness $k_{1}=5000 \mathrm{~N} / \mathrm{m}$, PTO damping coefficient $c=1000 \mathrm{Ns} / \mathrm{m}$, impact gaps $G_{1}=G_{2}=0.8 \mathrm{~m}$, wave height $H=0.8 \mathrm{~m}$, and causalisation time $t_{c}=3.2 \mathrm{~s}$.

The system matrices for the identified radiation subsystem in Eqs. (7) and (8) are

$$
\begin{aligned}
& A_{r}=\left[\begin{array}{l}
-1.50,-2.06,1.54,-0.35 \\
2.06,-0.01,0.07,-0.02 \\
-1.54,0.07,-2.38,1.96 \\
-0.35,0.02,-1.96,-0.54
\end{array}\right], \\
& B_{r}=[-403.88,22.57,-181.05,-49.82]^{\prime}, \\
& C_{r}=[-4.04,-0.23,1.81,-0.50] .
\end{aligned}
$$

The system matrices of the identified excitation force in Eqs. (12) and (13) are

$$
A_{e}=\left[\begin{array}{l}
-0.05,-0.61,-0.13,-0.24,-0.13,-0.12 \\
0.61,-0.19,-1.13,-0.29,-0.39,-0.24 \\
-0.13,1.13,-0.39,-1.56,-0.47,-0.52 \\
0.24,-0.29,1.56,-0.62,-2.04,-0.66 \\
-0.13,0.39,-0.47,2.04,-0.88,-2.38 \\
0.12,-0.24,0.52,-0.66,2.38,-1.21
\end{array}\right]
$$

$$
\begin{aligned}
& B_{e}=[-549.4,884.3,-1008.7,939.5,-784.6,603.3]^{\prime}, \\
& C_{e}=[-5.49,-8.84,-10.09,-9.39,-7.85,-6.03], \\
& D_{e}=[49.85] .
\end{aligned}
$$

\section{ACKNOWLEDGMENT}

This project has received funding from the European Union's Horizon 2020 research and innovation programme under the Marie Skłodowska-Curie grant agreement No 841388. This paper reflects only the authors' view and that the Agency is not responsible for any use that may be made of the information it contains.

\section{REFERENCES}

[1] S. H. Salter, "World progress in wave energy-1988," Int. J. of Ambient Energy, vol. 10, no. 1, pp. 3-24, 1989.

[2] M. Penalba, A. Ulazia, G. Ibarra-Berastegui, J. Ringwood, and J. Sáenz, "Wave energy resource variation off the west coast of ireland and its impact on realistic wave energy converters' power absorption," Appl. Energy, vol. 224, pp. 205-219, 2018.

[3] J. V. Ringwood, G. Bacelli, and F. Fusco, "Energy-maximizing control of wave-energy converters: The development of control system technology to optimize their operation," IEEE Control Syst. Mag., vol. 34, no. 5, pp. 30-55, 2014.

[4] J. H. Todalshaug, G. S. Ásgeirsson, E. Hjálmarsson, J. Maillet, P. Möller, P. Pires, M. Guérinel, and M. Lopes, "Tank testing of an inherently phase-controlled wave energy converter," Int. J. Mar. Energy, vol. 15, pp. 68-84, 2016.

[5] X. Zhang, J. Yang, and L. Xiao, "Numerical study of an oscillating wave energy converter with nonlinear snap-through power-take-off systems in regular waves," J. Ocean Wind Energy, vol. 1, no. 4, pp. 225-230, 2014.

[6] H. Bailey, "Influence of a nonlinear power take off on a wave energy converter," Ph.D. dissertation, $\mathrm{PhD}$ thesis, The University of Edinburgh, 2010.

[7] J. Sjolte, I. Bjerke, A. Crozier, G. Tjensvoll, and M. Molinas, "Allelectric wave energy power take off system with improved power quality at the grid connection point," in Proc. Transmission and Distribution Conference and Exposition, Orlando, USA, 2012, pp. 1-7.

[8] J. Henriques, L. Gato, J. Lemos, R. Gomes, and A. Falcão, "Peakpower control of a grid-integrated oscillating water column wave energy converter," Energy, vol. 109, pp. 378-390, 2016.

[9] A. Mérigaud and J. V. Ringwood, "Optimal trajectories, nonlinear models and constraints in wave energy device control," in Proc. 20th IFAC World Congress, Toulouse, France, 2017, pp. 15645-15650.

[10] M. Jaya Muliawan, Z. Gao, T. Moan, and A. Babarit, "Analysis of a two-body floating wave energy converter with particular focus on the effects of power take-off and mooring systems on energy capture," $J$. Offshore Mech. Arct., vol. 135, no. 3, 2013.

[11] A. Babarit and G. Delhommeau, "Theoretical and numerical aspects of the open source BEM solver NEMOH," in Proc. EWTEC, Nantes, France, 2015, pp. 6-11.

[12] B. Guo, R. Patton, S. Jin, J. Gilbert, and D. Parsons, "Nonlinear modeling and verification of a heaving point absorber for wave energy conversion," IEEE T. Sustain. Energ., vol. 9, no. 1, pp. 453-461, 2017.

[13] N. Faedo, Y. Peña-Sanchez, and J. V. Ringwood, "Finite-order hydrodynamic model determination for wave energy applications using moment-matching," Ocean Eng., vol. 163, pp. 251-263, 2018.

[14] J. Falnes, Ocean waves and oscillating systems: linear interactions including wave-energy extraction. Cambridge university press, 2002.

[15] B. Guo, R. Patton, and S. Jin, "Identification and validation of excitation force for a heaving point absorber wave energy convertor," in Proc. EWTEC, Cork, Ireland, 2017, pp. 1-9.

[16] B. Guo, R. J. Patton, S. Jin, and J. Lan, "Numerical and experimental studies of excitation force approximation for wave energy conversion," Renew. Energ, vol. 125, pp. 877-889, 2018. 